\title{
Monte Carlo Simulation-Based BEAMnrc Code of a 6 MV Photon Beam Produced by a Linear Accelerator (LINAC)
}

\author{
R. Sapundani ${ }^{1^{\star}}$, R. Ekawati ${ }^{2}$, K. M. Wibowo ${ }^{1}$ \\ ${ }^{I}$ Department of Electromedical Engineering, Universitas Muhammadiyah Purwokerto, \\ Jl. KH. Ahmad Dahlan, Kembaran Banyumas, Purwokerto 53182, Indonesia \\ ${ }^{2}$ Faculty of Information Science and Engineering, Jakarta Global University (JGU), \\ Jl. Raya Jatiwaringin No. 278, Jatiwaringin, Pondokgede, Bekasi 17411, Indonesia
}

\section{ARTICLE INFO}

Article history:

Received 22 April 2020

Received in revised form 14 August 2021

Accepted 18 August 2021

Keywords:

Monte Carlo simulation

Phase-space file

BEAMnrc code

DOSXYZnrc code

\begin{abstract}
A B S T R A C T
In radiotherapy, high energy ionizing radiation, such as X-rays, gamma rays and electron beams, is used. The dose in the tissue is often approached with the dose in the medium of the body which is $80 \%$ of human soft tissue. It is often difficult to determine the dose because the interaction of materials in a medium is very random. Measurement is also quite difficult because there are almost no detectors that are tissue equivalent. Measurement using an ion chamber requires a lot of correction to obtain a dose in the tissue, which is done using phantom and not directly in humans. This research aimed to compare the absorbed dose between modelling using Monte Carlo simulation and experiments. The simulation of dose distribution produced by a $6 \mathrm{MV}$ medical linear accelerator has been performed using BEAMnrc code running on Linux-based 2 processor system arranged in parallel. BEAMnrc was used to model and simulate the linac head with an SSD of $100 \mathrm{~cm}$ and Field size of $10 \times 10 \mathrm{~cm}^{2}$. A phase-space file is obtained as input to a DOSXYnrc code to produce Percent Depth Dose (PDD) in water and polymethyl methacrylate (PMMA) phantoms. New particles formed (electrons: $0.2 \%$, photon: $0.17 \%$; and positron: $0.08 \%$ ) were far from the contamination threshold of $2 \%$. The range of the correction factor of the depth of a maximum dose compared to the experimental data was 0.04-0.15.
\end{abstract}

(C) 2021 Atom Indonesia. All rights reserved

\section{INTRODUCTION}

Optimization of the therapeutic gain of radiation, i.e., maximizing the dose to tumours, while at the same time minimizing dose to healthy tissues, depends critically on the accuracy of the dose calculation [1]. Although the physics of radiation transport is very well known, analytical methods for dose calculations are associated with large errors, especially in patient's tissues where the effect of electron transport cannot be handled accurately. Measurement of the distribution dose is also quite difficult because there is no detector has the characteristics of tissue equivalent.

Monte Carlo algorithm, a physics principle for simulating radiation interactions in matter randomly, is an alternative treatment method used to determine

\footnotetext{
*Corresponding author.

E-mail address: tipung25@gmail.com

DOI: https://doi.org/10.17146/aij.2021.1046
}

the accurate dose. The method that takes into account these physical phenomena is very time consuming; meanwhile, in radiotherapy, time is a very strict factor because the treatment must be simulated and applied as quickly as possible [1,4].

The calculation of radiation doses in radiotherapy can be carried out by cross measurement between direct experiment and Monte Carlo simulation-based BEAMnrc and DOSXYZnrc code to obtain the more accurate results. BEAMnrc is used to model and simulate the linac head, whereas DOSXYZnrc code is used to design the phantom of the absorbed dose $[3,4]$. Both codes are developments from EGSnrc and can run on Linux/Unix platforms or Windows system. EGSnrc is user code for simulating radiation sources and modelling radiation transport (electron and photon) [1-4]. The simulation dose profiles when compared to the measured data, using the gamma index 
method, were in good agreement with a correction factor of $3 \%$. [4].

This paper reports the simulation of depthdose distribution for $6 \mathrm{MV}$ photon beam on a LINAC using Monte Carlo-based BEAMnrc Code with an SSD of $100 \mathrm{~cm}$ and field size of $10 \mathrm{~cm} \times 10 \mathrm{~cm}$. The PDD was derived from the DOSXYZnrc code simulation, which was then compared with the measured data. Using parallel computer, Monte Carlo calculations with the EGSnrc system can be carried out easily and quickly. In this case, a single simulation can be executed simultaneously using more than one Linux-based computer so that it is more time saving in performing the simulation as a whole: controlling the simulation on each computer, observing how much history has been simulated and how much not. If all the history has been completed, it is simulated, where each output file from each computer is merged into one final output file.

\section{EXPERIMENTAL METHODS}

\section{Configuration of linear accelerator}

There are several configurations to perform exposure with medical linear accelerators: type of exposure, energy (top), field size, exposure (field size) in the phantom surface, collimator angle, gantry position, and source surface distance (SSD). The exposure used in this study was an open-beam photon (without the wedge and other accessories) with the energy of $6 \mathrm{MV}$ and an exposure field size of $10 \mathrm{~cm} \mathrm{x} 10 \mathrm{~cm}$ at the phantom surface. The collimator angle for this configuration was zero degrees. The gantry position was set perpendicular to the phantom. The focal point distance of the radiation source to the phantom surface (SSD) was fixed to $100 \mathrm{~cm}$, as can be seen in Fig. 1. [5,6].

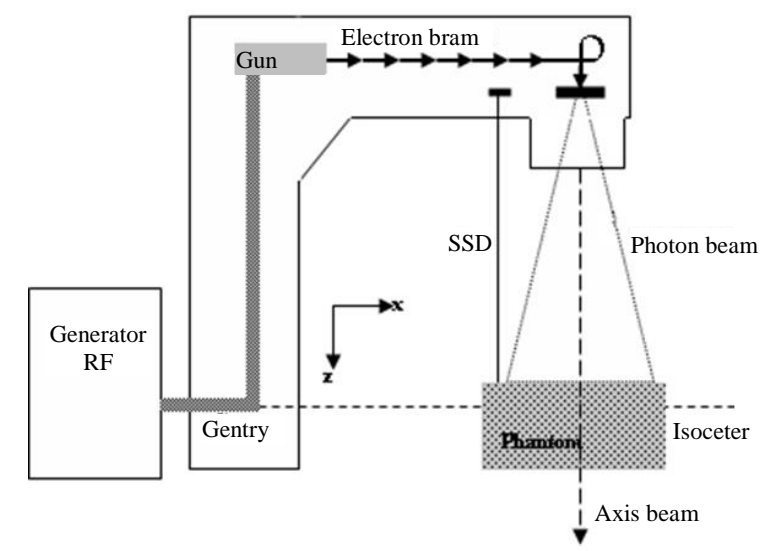

Fig. 1. Configuration Exposure for Linear Accelerator.

The phantom design was built from the composition of a homogeneous slab phantom (PTWtype RW3 2967). The configuration of exposure for medical linear accelerators was modelled using RTP system at Gatot Subroto Hospital (RSPAD).

\section{Percentage Depth Dose (PDD)}

The instruments to measure PDD were 3D Water Phantom (PTW-MP3 Water Phantom), a detector (waterproof semiflex ionization chamber from PTW with an effective volume of $0.6 \mathrm{~cm} 3$ ), and a dosimeter (from PTW). The dosimeter is connected to a PC (Windows OS) that is equipped with MEPHYSTO software as a display and controller for the detector adjustment process to regulate the movement pattern of the detector in phantom [7].

The absorbed dose varied with depth when a material (phantom) was radiated. The difference absorbed dose depends on several conditions, including energy, depth, size of exposure field, and calculation of the distance between points dose (SSD or SAD) at the phantom from the radiation source. One way to characterize the dose distribution $(\%)$ is to normalize the dose at each depth with a dose at a depth that is used as a reference (reference depth). Reference depth that can be used in radiotherapy with low energy $(\leq 999 \mathrm{keV})$ is the phantom surface. For high energies, the reference depth can use PDD using Eq. (1).

$$
P D D=\frac{\text { Certain depth dose }}{\text { Reference dept dose }} \times 100 \%
$$

\section{Profile dose}

The relationship between dosage on the main axis and its outside is very complicated, depending on radiation energy, SSD, collimator properties, field size, the angle between the main axis of the radiation beam and the surface of the medium [8], block that is forming field, source size, penumbra [9], symmetry, uniformity of radiation beam, flattening filter [10]. As a result of the numerous factors that influence dosage, not all of the doses outside the central axis can be expressed by a mathematical formula. This means that the outlier doses must be measured directly to obtain their value. In this study, a dose profile at a certain depth was used to illustrate doses outside the central axis. The dose profile is influenced by three parameters, namely symmetry, beam surface (flatness) and penumbra [10].

\section{Monte Carlo simulation}

The linear accelerator (LINAC) in this paper is described in the perspective of Monte Carlo-based 
EGSnrc that models photon and electron transport. The design was done using BEAMnrc protocol developed by the National Research Council of Canada (NRCC). The first step in modelling the accelerator was to specify accelerators by selecting components to be used. The LINAC head components built by BEAMnrc includes the target, primary collimators, flattening filter, ion chamber, mirror, a secondary collimator (Y jaws and $\mathrm{X}$ jaws), Fig. 2. [11,12].

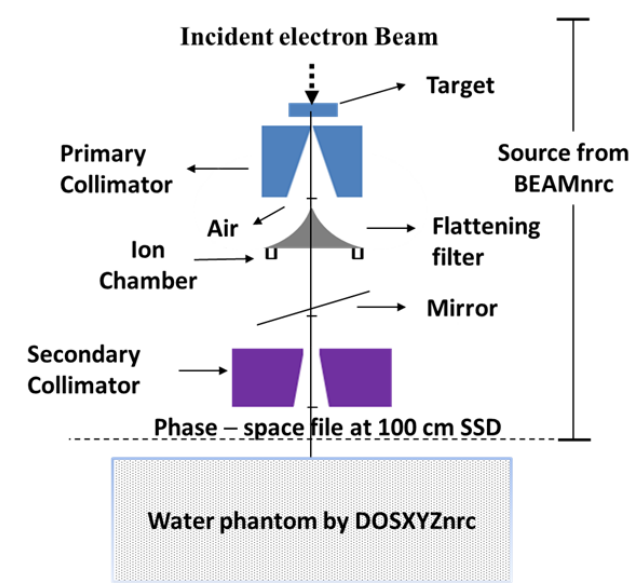

Fig. 2. Preview of linac head design using BEAMnrc.

After the components are determined, the model can then be compiled. The next step was to enter data related to the geometry of the accelerator, incoming particles, and scattering cross-section data associated with the process of particle interactions that would occur in an accelerator.

To do the simulation, the user can execute the file compilation (binary/executable files) with two pieces of information provided previously, namely the file.egsinp and file.pegs4dat. Files.egsinp is read to determine the geometry parameters, material type, EGSnrc (physics events to be calculated and considered) and Monte Carlo Material data, which are selected based on type. Egsinp will read from the directory file.pegs4dat pegs4. The simulation results using BEAMnrc can consist of data about the phase space (phase-space file), listing the output files, and graphics files. Graphics files can be presented using graphics packages EGS_Window, Fi. 3. [2,13].

Phantom modelling aims to investigate the PDD (percentage depth dose) or radiation dose from the accelerator head at a certain depth. Phantom is irradiated by a specific source, modelled by DOSXYZnrc by reading the file space files, Egsinp and Pegs4dat $[14,15]$.

Simulations on phantom were divided into two parts. First, the depth dose was simulated by entering the phase space data at the bottom of the head of the accelerator jaws, i.e., chamber components. The simulation was carried out in the chamber with the main materials of air and the PMMA. The second simulation was to determine the depth dose in a water phantom using the DOSXYZ program with phase space data as a source. After normalizati on of output data (phase-space file as input) is done, the PDD is obtained. Further simulation was carried out using BEAMnrc on the water phantom.

\section{Radiation beam of photons and electrons}

In the BEAM system, the variable Latch (Non-Inherited Latch) was used to determine the amount of contamination in the accelerator head components as indicated by the emergence of new particles resulting from photon beams interaction with the accelerator component materials. Transport parameters of particles in the medium are photon forcing, range rejection and Bremsstrahlung-uniform splitting as a variance reduction. A couple of seed random numbers used at the beginning of the simulation were 31 and 77 . For all stages of modeling, the electron cutoff energy and photon cutoff energy were each set at $0.7 \mathrm{MeV}$ and $0.01 \mathrm{MeV}$ [16-18].

\section{Parallel computing}

To shorten the computing time, concerning the amount of data collection that could reach $10^{8}$ incidents or events, a computer with a highspeed processor was used. Linux-based machines were used to run the Monte Carlo simulation. The machines has high efficiency and has an overall reduction of computing time when compared with Microsoft Windows-based machines. The next efficiency step was to use more than one PC, all of which were arranged parallel to each other, as shown in Table 1 [19-21]. The software used to run in parallel was Sun Grid Engine (Grid cluster) [22].

Table 1. Specification of Computers in simulation

\begin{tabular}{cc}
\hline Memory & Processor \\
\hline $1 \mathrm{~GB}$ & Pentium 4 (3 GB) \\
$760 \mathrm{MB}$ & AMD I $(3.2 \mathrm{~GB})$ \\
\hline
\end{tabular}

\section{RESULTS AND DISCUSSION}

\section{Photon and particles contamination}

Variable Latch (BEAM system) indicates that contamination of new particles formed is far from the contamination threshold of $2 \%$ 
(electrons: $0.2 \%$, photon: $0.17 \%$; and positron: $0.08 \%)$. Based on previous findings, simulating $6 \mathrm{MV}$ photon beam as the MC methods indicates that contribution of electrons is less than $3 \%$ of maximum total dose for $10 \times 10 \mathrm{~cm}^{2}$ field sizes [12]. Other previous study running on $12 \mathrm{MV}$ states that the electron contamination giving contribution in surface dose is less than $1 \%$ for field size $10 \times 10 \mathrm{~cm}^{2}$ [13].

\section{Comparison of PDD}

To ensure the reliability of the Monte Carlo simulation's validity, a comparison was carried out between the PDD graphics based on Monte Carlo modelling and the experiment data, as shown in Fig. 4 and Table 2. The depth of maximum doses between the modeling and the experimental results has relatively no differences at a range of correction factor of 0.04 to 0.15 . A study of high-energy linear accelerator (10 MV) performed by Yani [13] shows that the differences are less than $2 \%$. The measurement performed by $\mathrm{S}$. Didi for the Tomographic Emission Monte Carlo software shows that the dose differences is about $0.02 \%$ [8].

Table 2. Depth of max. doses between modelling and experimental.

\begin{tabular}{cccc}
\hline Photon & Material & $\begin{array}{c}\text { Depth of } \\
\text { max. Dose }\end{array}$ & $\begin{array}{c}\text { range of } \\
\text { correction factor }\end{array}$ \\
\hline & Water (BEAMnrc) & 1.4 & $0.15 \%$ \\
6MV & PMMA (BEAMnrc) & 1.4 & $0.15 \%$ \\
$\begin{array}{c}\text { simula } \\
\text { tion) }\end{array}$ & Water (DOSXYZ) & 1.7 & $0.04 \%$ \\
& $\begin{array}{c}\text { Air-fine, 1 mm } \\
\text { (BEAMnrc) }\end{array}$ & 1.5 & $0.09 \%$ \\
\hline & $6 \mathrm{MV} \mathrm{(Exp.)}$ & 1.6 & \\
\hline
\end{tabular}

Table 3. Relative dose to Exp. (\%).

\begin{tabular}{lccccc}
\hline \multirow{1}{*}{ Material } & \multicolumn{5}{c}{ Depth (cm) } \\
\cline { 2 - 6 } & 18 & 20 & 22 & 25 & 28 \\
\hline Water (BEAMnrc) & 0.4 & 0.3 & 0 & 3 & 3 \\
PMMA (BEAMnrc) & 8 & 8 & 12 & 13 & 14 \\
mm ( air-fine, & 0.7 & 0.5 & 1 & 2 & 4 \\
BEAMnrc) & 1 & 1 & 2 & 3 & 3 \\
DOSxyz & & & &
\end{tabular}

The relative dose (\%), except for PMMA, between the results of the Monte Carlo simulation and the measurement data is below $3 \%$ with a depth of $20 \mathrm{~cm}$, whereas for depths greater than $20 \mathrm{~cm}$ is below $5 \%$, as shown in Table 3 . In this case, PDD of PMMA is lower than water phantom (soft tissue equivalent). Thus it can be said that the model created in this simulation is still quite valid.

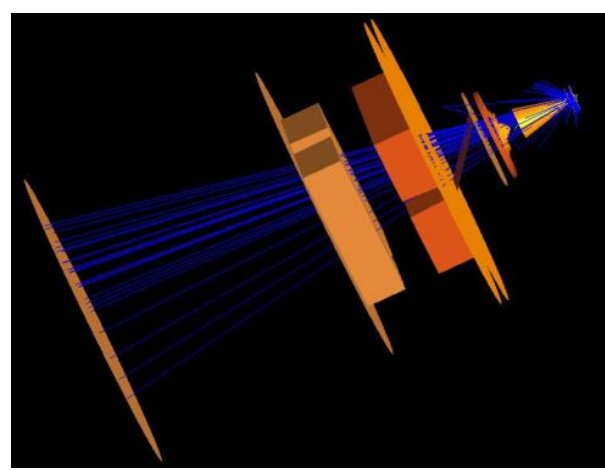

Fig. 3. 3-D LINAC head using EGS_Windows (BEAMnrc).

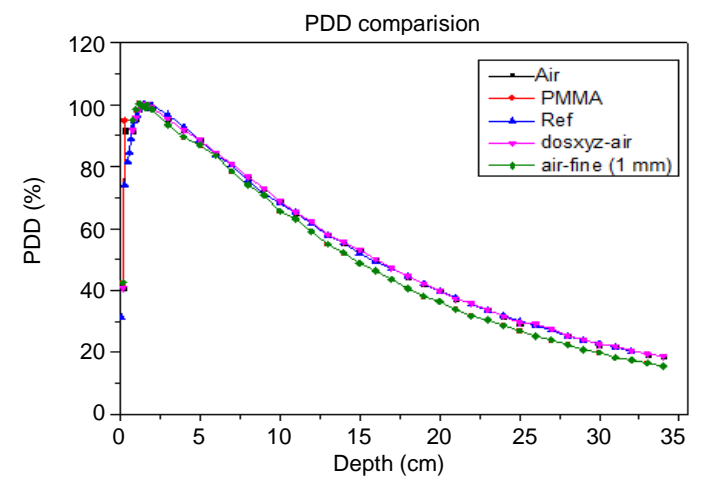

Fig. 4. PDD Comparison between modelling result and experiment.

Figure 5. is an enlargement of Fig. 3 at $5 \mathrm{~cm}$ depth. Electronic equilibrium has not been reached when measuring at lower depths up to Dmax (covering the build-up areas). This causes the results of the chamber experiment to be less reliable. Both ideal measurements on small $\mathrm{z}$ should use a dot detector which has a very small size. Such a detector is rare in the markets except for diamond detectors that are very costly.

Monte Carlo has a comparative advantage because it comes from the calculation of "virtually ideal" probabilities. Thus we can speculate that in Figs. $4 \& 5$ the results of Monte Carlo are more "satisfying" in comparison to the experimental measurements.

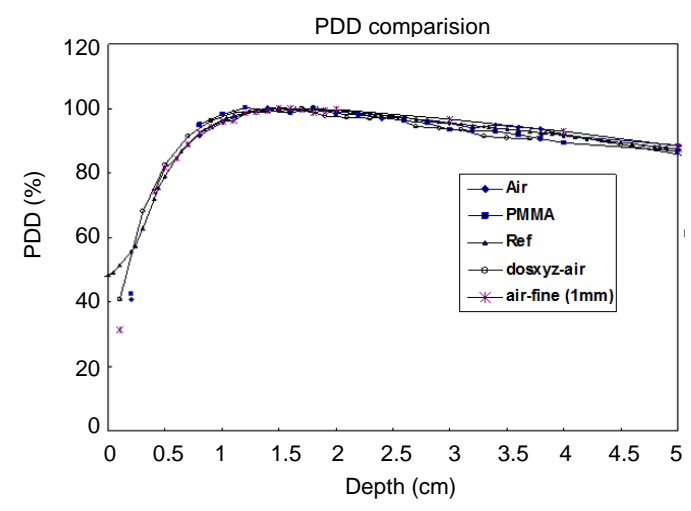

Fig. 5. PDD comparison from the phantom surface to in-depth of $5 \mathrm{~cm}$. 


\section{Dose profile}

Radiation technique is very important because it relates to the dose distribution in tumours. Through good radiation techniques, dose distribution in the tumour can be distributed evenly and higher than in the healthy tissue. Figure 5 shows the profile dose of the simulation and the experimental data, which explains the large number of doses and field size required in radiotherapy treatment.

Penumbra widths of the dose profile's right area are relatively equal, approximately $0.43 \mathrm{~cm}$. Figure 6 shows the beam symmetry and surface beam for the dose profile of the experiment results and the simulation, which are relatively similar and coincidental. Small differences in these results are constant and reproducible despite many times repetition.

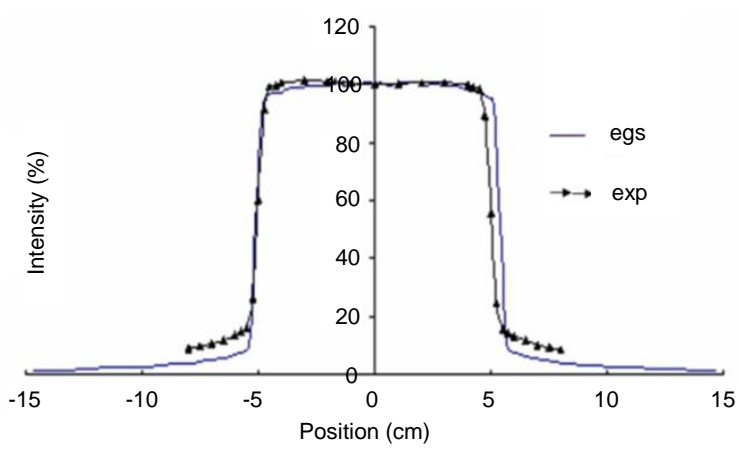

Fig. 6. Dose profile comparison between simulation and experiment in the depth of $5 \mathrm{~cm}$.

\section{CONCLUSION}

PDD comparison between modelling and experiments has no significant differences, i.e., 0.02 $\neg 0.15$ (\% correction). There are only differences in the peak dose locations. Penumbra width, beam symmetry and surface beam for dose profile from the experiment result are relatively similar and coincidental. One of the possible differences is from the ideal condition of the geometric LINAC or the initial set of parameters in the BEAMnrc code. This requires further research. Results of the LINAC head model prove that the Monte Carlo method with BEAMnrc code is accurate enough to model software of linear accelerator heads starting from the energy source until the stage of modelling phantoms for dose measurement.

\section{ACKNOWLEDGEMENT}

We would like to express our gratitude to Dr. Rachmat Widodo Adi for his support and thoughtful guidance.

\section{AUTHOR CONTRIBUTION}

Rum Sapundani and Kusnanto Mukti Wibowo wrote the manuscript. Rum Sapundani carried out the experiment by running Monte Carlo simulationbased BEAMnrc and DOSXYZnrc code. Rum Sapundani and Ris Ekawati built the paralel personal computer.

\section{REFERENCES}

1. M. Machichi, Y. Oulhouq, A. Rrhioua et al., Mater. Today Proc. 13 (2019) 982.

2. D. W. O. Rogers, B. Walters and I. Kawrakow, BEAMnrc Users Manual, NRCC Report PIRS-0509(A)revL, Canada (2021).

3. S. Gholampourkashi, J. E. Cygler, J. Belec et al., E. Heath, J. Appl. Clin. Med. Phys. 20 (2019) 55.

4. M. Bencheikh, A. Maghnouj, J. Tajmouati et al., Phys. Part. Nucl. Lett. 14 (2017) 780.

5. J. EL Bakkali and T. EL Bardouni, J. King Saud Univ. - Sci. 29 (2017) 106.

6. R. Shende, G. Gupta, G. Patel et al., Int. J. Med. Physics, Clin. Eng. Radiat. Oncol. 05 (2016) 51.

7. R. N. Sruti, M. M. Islam, M. M. Rana et al., Nucl. Sci. Appl. 24 (2015) 29.

8. S. Didi, A. Moussa, T. Yahya et al., J. Med. Phys. 40 (2015) 136.

9. B. Kadman, N. Chawapun, S. Ua-Apisitwong et al., J. Phys.: Conf. Ser. 694 (2016) 1.

10. K. R. Mani, M. A. Bhuiyan, M. S. Rahman et al., Polish J. Med. Phys. Eng. 24 (2018) 79.

11. M. Mohammed, T. El Bardouni, E. Chakir et al., J. King Saud Univ. - Sci. 30 (2018) 537.

12. A. Kajaria, N. Sharma, S. Sharma et al., Int. J. Appl. Eng. Res. 11 (2016) 8185.

13. S. Yani, M. F. Rhani, R. C. X. Soh et al., Int. J. Radiat. Res. 15 (2017) 275.

14. K. Sachse and F. C. P. du Plessis, Phys. Medica 41 (2017) 3.

15. K. Hadad, M. Saeedi-Moghadam and B. Zeinali-Rafsanjani, Technol. Health Care 25 (2017) 29.

16. A. Ghila, S. Steciw, B. G. Fallone et al., Med. Phys. 44 (2017) 4804.

17. B. R. B. Walters, Med. Phys. 42 (2015) 5817.

18. J. E. Morales, M. Butson, R. Hill et al., Phys. Eng. Sci. Med. 43 (2020) 609. 
19. S. Stathakis, F. Balbi, A. T. Chronopoulos et al., J. BUON. 21 (2016) 252.

20. M. Nasir, D. Pratama, C. Anam et al., J. Phys: Conf. Ser. 694 (2016) 1.

21. L. T. Campos, L. A. Magalhães and C. E. V. de Almeida, J. Biomed. Phys. Eng. 9 (2019) 259.
22. S. Bao, F. D. Weitendorf, A. J. Plassard et al., Theoretical and Empirical Comparison of Big Data Image Processing with Apache Hadoop and Sun Grid Engine, Proceedings, Medical Imaging, Imaging Informatics for Healthcare, Research, and Applications 10138 (2017) 101380. 